\title{
TiMBA - Tangible User Interface for Model Building and Analysis
}

\author{
Chih-Pin Hsiao ${ }^{1}$ and Brian R. Johnson ${ }^{2}$ \\ ${ }^{1}$ College of Architecture, Georgia Institute of Technology, Georgia, USA \\ ${ }^{2}$ DMG Lab, College of Built Environments, University of Washington, Washington, USA \\ chsiao9@gatech.edu, brj@uw.edu
}

\begin{abstract}
Designers in architectural studios, both in education and practice, have worked to integrate digital and physical media ever since they began to utilize digital tools in the design process [1]. Throughout the design process there are significant benefits of working in the digital domain as well as benefits of working physically; confronting architects with a difficult choice. We believe emerging strategies for human-computer interaction such as tangible user interfaces and computer vision techniques present new possibilities for manipulating architectural designs. These technologies can help bridge between the digital and physical worlds. In this paper, we discuss some of these technologies, analyzes several current design challenges and present a prototype that illustrates ways in which a broader approach to human-computer interaction might resolve the problem. The ultimate goal of breaking down the boundary between the digital and physical design platforms is to create a unified domain of "continuous thought" for all design activities.
\end{abstract}

Keywords: Tangible User Interfaces, Computer Vision, Architectural Design.

\section{Introduction}

Design is a challenging cognitive activity, involving tactile, spatial, and auditory perception and "wicked problems" [2]. Buildings are almost invariably unique, inappropriate on another site, in another climate, or with other functionality and requirements. Architects must often address conflicting goals using limited information, and seeking a solution that harmonizes the parts in a process sometimes referred to as "puzzle making" [3]. The process is complex, cyclical and requires concentration, making it prone to disruption from external sources as well as internal ones.

During the process, architects use external media to record and explore their designs. Traditionally, pen and paper were used for drawings, and cardboard, wood, clay and other materials were used for models. While most work is now done on computers using CAD and modeling software, architects in the early stages of design are often more comfortable directly creating or modifying hand drawings and concept models than using software. A model may be used directly to study physical issues such as shadowing and lighting; it can be used as the focus of face-to-face conversations with clients who might have difficulty interpreting architectural drawings and digital models, and the designer can trim or replace parts in order to 
study alternatives. This does not mean they are unaware of the value of digital tools. Digital models are known to be useful for high quality visualization, extraction of coordinated drawings and cost estimates, and key to energy and lighting simulations, etc. Unfortunately, the issues are often quite subtle, the typical "direct manipulation" interface [4] provides limited freedom for designers to study their designs, and it remains difficult to edit in 3D on a 2D screen [5].

The apparent need to choose between two high-value tool sets motivates this vision: your computer 'partner' watches over your shoulder as you construct a cardboard model and simultaneously builds a digital model. You would have the physical artifact and the digital data at no extra cost. In fact, the two might be used together to provide even greater insight into the design as it is being developed.

\section{Related Work}

In 1997 Hiroshi Ishii proposed Tangible User Interfaces (TUIs) as a new way to bridge between "Bits and Atoms" [6]. He argued that information "bits" would come into the real physical world through everyday physical objects and environments (atoms) [6]. By making digital information tangible, he thought the gap between cyberspace and physical environments could be bridged. During design, spatial cognition plays a very important role, helping us understand complex relationships and manipulate abstract concepts. Interruptions can disrupt continuity of thought and complex GUIs frequently present command-selection interruptions. However, Tangible User Interfaces may enable us to better apply our intelligence during the design process.

There have been several previous efforts to create tangible interfaces for design processes. In "URP", Underkoffler and Ishii address the issue in the context of urban planning by combing tangible models and projected simulation data [7].

There is other research that addresses the relationship between the physical and digital geometries by using smart materials. Construction kits, such as LEGO $^{\mathrm{TM}}$ Technic, are easy to use [8]. Anderson et al. made a project that combines building blocks and a scanning system to achieve the goal of modeling and reducing the inaccessibility of the traditional sketch modeling system. "FlexM" is another project that uses two kinds of construction kits to build and shape a 3D digital model [9]. Researchers developing "Posey" utilize embedded sensors to make construction kits more complicated and adaptable to design [10]. Instead of using smart materials, Song et al. use digital pen for recording annotation and editing geometry in both physical and digital world simultaneously [11]. However, in these systems, the digital geometries have to be pre-built and registered to the particular physical objects, which is not desirable for designers in the midst of the design process.

\section{The TiMBA Prototype}

\subsection{System Design Considerations}

Cardboard models are commonly used by architects in studying the form of a building. To construct a digital model from a physical one, our "over the shoulder" 
software system has to know the shape, the location and the orientation of the individual pieces of cardboard. There are several possible ways to find the whereabouts of physical objects for the system, such as embedding radio frequency ID tags in every piece of cardboard, building a model with multiple 6 degree-offreedom sensors, or detecting the related position of every piece by knowing which are attached, such as the improving version of Posey [10]. However, these all restrict the range of formal expression available to the designer. In the end, we adopted a computer vision strategy in order to work with almost any cardboard, permit reshaping of pieces, and minimize instrumentation costs (as compared to processing time) while producing a practical system. We divided the model-building process into two steps. The first step employs an edge detection algorithm to define the shape of each cardboard piece. The second step uses the marker-tracking library from ARToolKit [12] to locate each cardboard piece in the physical assembly.

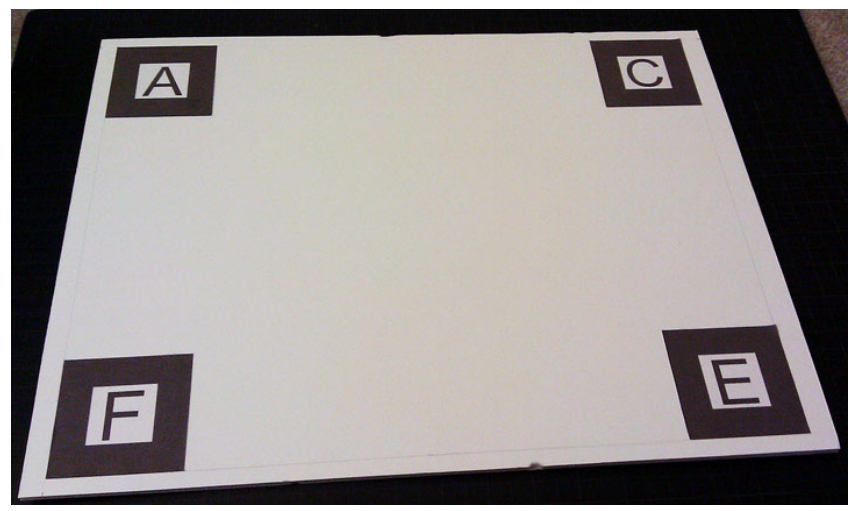

Fig. 1. Foundation board for users to build model on

Since cardboard pieces are usually cut flat in the first place, we provide a black cutting mat with an overhead camera for a better edge detection process. After cutting, each piece is given a fiducial marker. The camera acquires an orthogonal, or "true-view" of the piece and links it to the marker ID and location on the piece. After users combine the individual cardboard pieces in step two, the composite digital model can be computed using the positions of the markers relative to a marker set on the foundation board (Figure 1). As the designer works, a digital model is constructed from their physical model. If the designer decides to reshape a piece, they only need to remove it from the model and adjust the size on the cutting mat before re-inserting it in the composition.

\subsection{System Overview}

In this prototype, we modified ARToolKit 2.7 (ARTK) to address both vision tasks, and used an API for the commercial modeling program Google SketchUp to build the host-modeling environment. ARTK is designed for use in Augmented Reality applications. TiMBA utilizes the ARTK edge-detection code and the ability to register the location and orientation of fiducial markers. Two applications based on 
ARTK were created to complete the two steps identified above. Step 1 is controlled by the "Shape Scanning Application" (Figure 3) and Step 2 is controlled by the "Location Detector Application" (Figure 4). Each application runs continuously, receiving frames from its respective camera and performing its analysis. They transfer results to a custom script running in SketchUp, which combines the results. Our SketchUp script, "SketchUp Ruby Helper" (SRH), receives the data provided from the two ARTK applications and uses it to control the shape, location, and orientation of the geometry of the finished digital model. Figure 2 shows the whole system architecture.

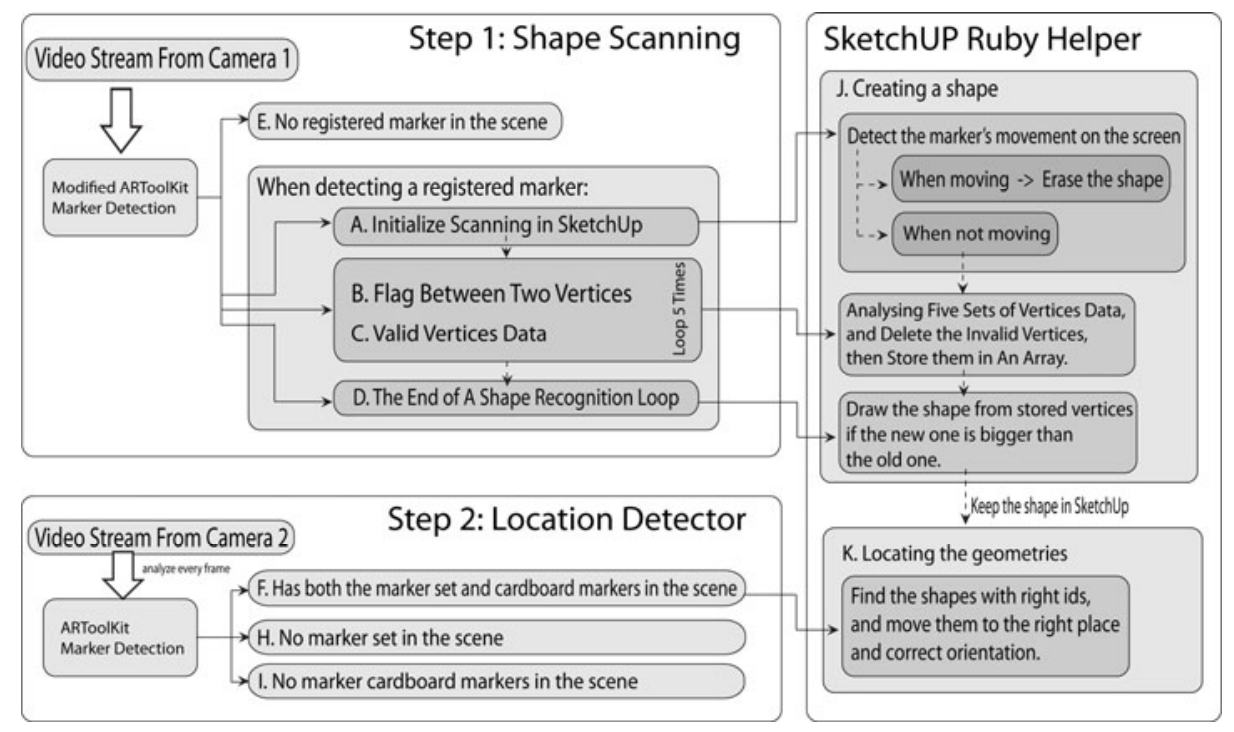

Fig. 2. The System Architecture of TiMBA

\subsection{Step 1 - The Shape Scanning Application (SSA)}

The Shape Scanning Application (SSA) uses a utility process from ARTK to accumulate the dark area, and gives the same area a flag. In the original ARTK process, these are recognized whether they are a four-vertex shape or not. In our modified version, the new code grabs the area information of reversed color to recognize the number of vertices around the contour of the area and which pixels these vertices are when the previous frame contains a fiducial marker. In order to get more accurate vertex information, TiMBA combines information from a number of video frames.

In addition to the vertex information, SRH needs to know the size of the shape, the ID of the marker, and the transformation matrix of the marker. These data, combined with the vertex data will comprise an entry in the complete data stream. A Unix "pipe" is used to communicate between SSA and SRH. Each cardboard piece is represented as a record similar to the following: 
Table 1. Sample record of data transferred from SSA to SRH

\begin{tabular}{c|c|c|c|c|c|c|c|c|c|c|c}
-1 & 2 & 383.7 & 340.1 & 170.2 & 277.7 & 164.5 & 197.9 & 268.8 & 187.6 & 283.2 & 264.1 \\
\hline $\mathrm{a}$ & $\mathrm{b}$ & $\mathrm{c}$ & $\mathrm{d}$ & $\mathrm{e}$ & $\mathrm{f}$ & $\mathrm{g}$ & $\mathrm{h}$ & $\mathrm{i}$ & $\mathrm{j}$ & $\mathrm{k}$ & $\mathrm{l}$
\end{tabular}

In Table 1, the meanings of these numbers are as follows: (a) beginning of data flag, (b) the marker id, (c) marker center X, (d) marker center Y, (e) to (l) the coordinates of the four vertices of the marker on the XY plane of the piece of cardboard.

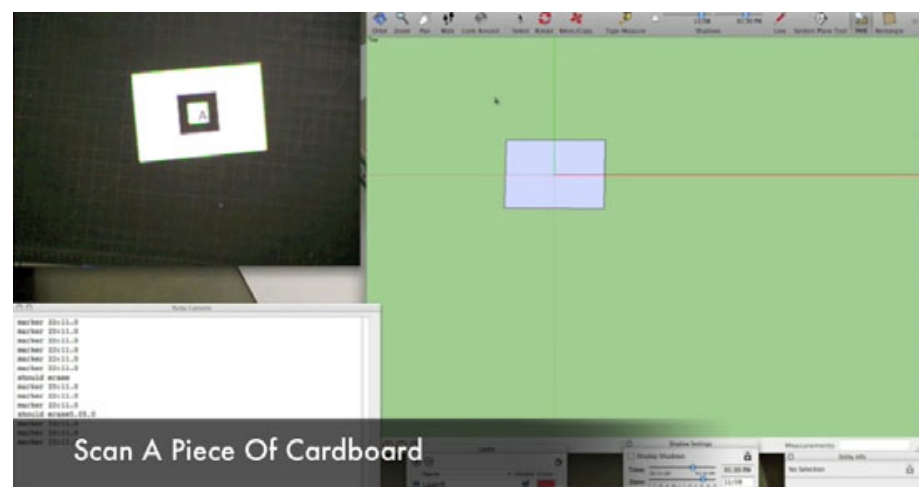

Fig. 3. The Screen Shot in Step 1

\subsection{Step 2 - The Location Detector Application (LDA)}

The concept of this application is simply to compare the location data of the cardboard markers to the coordinate system provided by a "base" marker set on the foundation board (Figure 1). Results are transferred to SRH to drive the model display. To improve the speed when multiple markers are encountered in the same scene, a continuity assumption is made: in the absence of contraindication, parts do not move. For each frame analyzed, the system compares the location data of the markers it sees to that of the previous frame. If a marker has not moved too much, it will not send new location information for the marker, but send the next moving marker in the current loop instead.

\subsection{The SketchUp Ruby Helper (SRH)}

This program has to analyze the data from both LDA and SSA. Then, it transforms them into the coordinate system of SketchUp and displays the geometry for users. There are three main tasks to deal with in this application: 1) creating a 2D shape, 2) moving the $2 \mathrm{D}$ geometry to the appropriate $3 \mathrm{D}$ location, and 3) trying to glue the adjacent shapes together. The first two tasks simply take the data from the other two applications and analyze them to verify a reasonable shape and location. The goal of the third task is to assert physical continuity in the system - to assemble the cardboard 
pieces as one big model, seeking to avoid the situation where, because the camera for the LDA cannot see a marker at the rear of the model but the user's viewpoint shows it, pieces of the digital model "drop out".

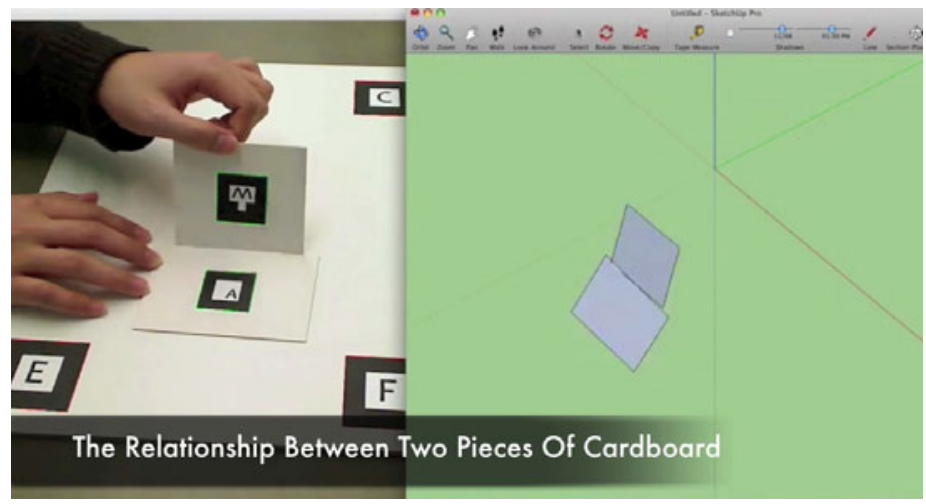

Fig. 4. The Screen Shot in Step 2

\subsection{Discussion}

This version of TiMBA helps the user make a digital model by "observing" their construction of a physical model. It can reflect the movement, shape, and position of a piece of cardboard in the physical world and give some response back to the user. However, the real benefits of this kind of system reside in the synergy that might be achieved between the physical and the digital by utilizing the ability to give much more information and have a better way to represent the information than the physical model can provide. Before this happens, we believe that there are some improvements we need to make. Even though the prototype provides real time information from the physical side, response-time is inadequate. In SSA, after the camera sees a marker, it takes a while to "grow up" the right shape in SketchUp. Likewise, in LDA, the movement of the digital geometry, driven by movement of the physical cardboard, is not smooth enough. Accuracy is another issue. Since the system has to run in real time, the result of the computer vision analysis is not always as accurate as in a static scene. Although TiMBA includes efforts to mitigate this error by "gluing" or linking nearby edges from two different shapes, this issue would still be an irritant to users. Self-occlusion is another issue alluded to previously. The physical model is opaque. As pieces are added, they may obscure the camera's view of other pieces. Currently we rotate the model base to enable the camera to see all of the model. An additional camera view of the model would enable the system to run more smoothly, but selfocclusion remains an issue. While imperfect, we believe that this prototype is just the beginning, and there are still many more possibilities we could explore.

\section{Preliminary Experiment}

Observations of users are always valuable because everyone has his/her own habits when building models. Therefore, after we built the prototype, we asked several 
students from our architecture school to test it. While TiMBA uses a general modelbuilding approach intended to fit every designer's habits, we had to add AR marker placement and scanning to the workflow. Further, we wished to test the utility of the system to help users answer certain architectural questions through modeling.

At the beginning of the tests, the participants were given a short set of instructions and permitted a 5 minute "learning phase" so that they could familiarize themselves with the system. We provided the same tools and materials for each participant to complete both a digital and a physical model using TiMBA. After finishing the learning phase, they were given two tasks, including the building of digital and physical models by provided drawings and the answers to two related questions. For these tasks, the subjects were asked to consider a particular design of an element for this building. We also avoided discrete, binary, yes/no style answers for tasks, which might encourage guessing. Subjects were told they were being timed on the experiment and to "complete each task as quickly as possible." After building the models, they were asked to save the digital model and kept the physical one for the experiment result, and then moved on to the next experimental condition. At the end, they were given a post-experiment questionnaire.

We employed both performance measures and subjective perceptual measures. In performance measures, we recorded a) time (the number of minutes to complete the test) and b) the accuracy of the results. Assessing accuracy, we compared their answers to the task questions with a correct answer. For example, the first task is "at 12:00 pm, June 21, which walls would receive direct sunshine (east, west, south, north)?" The subject had to build an accurate model and then use the digital model to simulate the shadow patterns on the site. An alternative question was "which orientation of the building is the best one in terms of avoiding the shadows from the other building near the site for the garden near the building (east, west, south, north)?" In this case, the subject could rotate the digital model until they achieved a satisfactory answer. The results are convincing. All of our subjects achieved the correct answers in a relatively short time when constructing both of the digital and physical models.

Figure 5 shows the list of questions and the results from the post-experiment questions. In general, the subjects did not think "sticking the marker" on the pieces distracted them from building the physical model. They also believed that the assistance from the computer helped them answer questions about the design. However, there are still some features that bother users: the prototype requires users to scan each piece of cardboard before assembling them, a process which takes several seconds. This annoyed subjects who are used to gluing two pieces of cardboard together right after cutting them. In addition, users must pay attention to which side of the cardboard they affix the AR marker to, because the system needs to see the marker to locate the geometry. They also think the prototype could be more intuitive. According to the observation, the separation between the working and data representation platforms could cause the intuitive issue with which the subjects encountered. Although they thought they could build a better digital model with mouse and keyboard, some of them indicated that it depends on the complexity of model. For instance, some complicated models require advanced digital modeling skill. On the 
physical side, however, we only need our hands to bend materials or place them at the specific location. Overall, they think this is a fun and effective way to build a digital model.

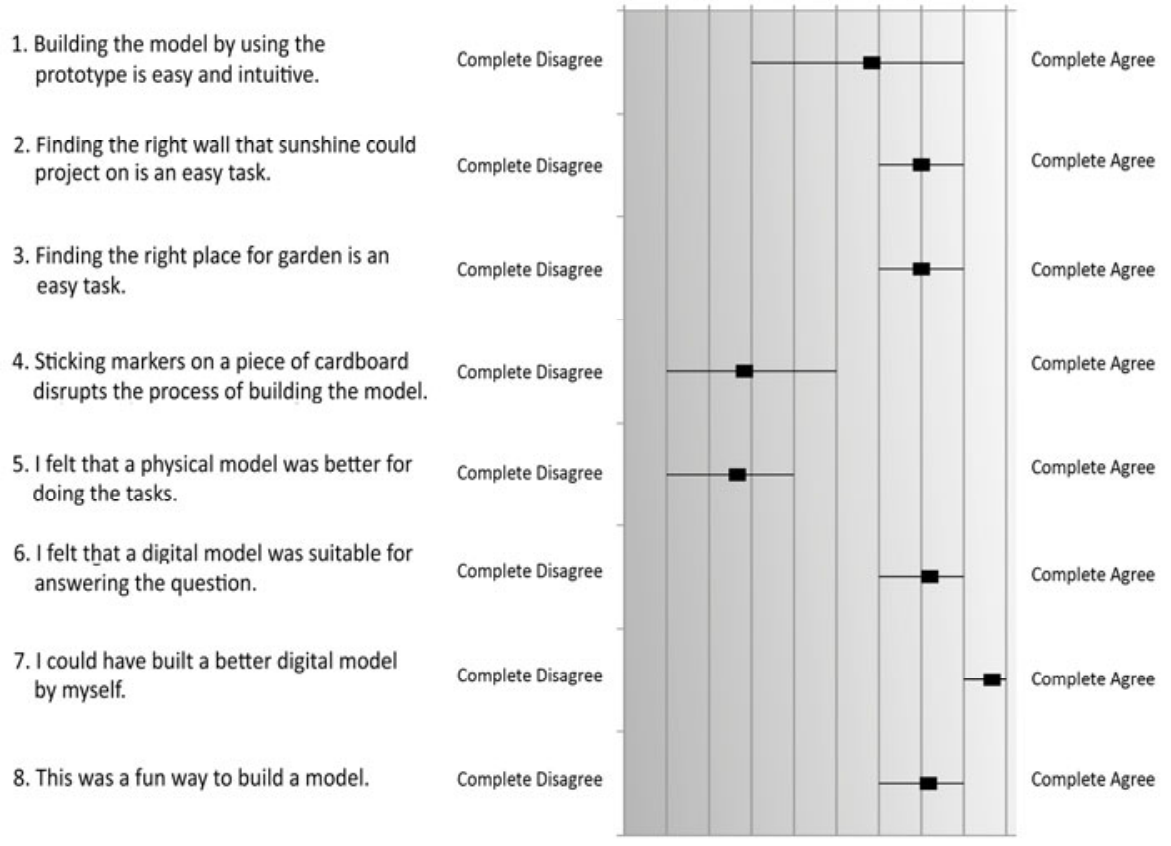

Fig. 5. List of the Assessment Questions and the Results

\section{Conclusion}

In this paper we have presented some recent uses of Tangible User Interfaces and other digital technologies, how they are increasingly important in the design process, and how, by using these new technologies, we may derive certain benefits. We also explained why we believe designers will not totally abandon the traditional design media, which rely on hand drawings and physical models. Finally, we described TiMBA, a prototype software system that we developed to demonstrate that the affordances of both physical and digital workflows might be combined synergistically. The most exciting "take-away" from these applications is the notion that we can treat a physical object in digital ways. This could lead to infinite possibilities when computational power can get "off the screen" and lay on our working platform.

Why do we not simply finish all the tasks in a virtual world? Why do we need to connect the physical and virtual worlds together? We believe that the ubiquity of computational power will let us treat computational tools as additional physical objects. These two different categories of "objects" are essentially the same thing, 
created and utilized by us. We seek to make available the affordances of every tool designers wish to utilize to achieve their goals. The computational power is just the magic that can make these objects alive and offer them the ability to interact with humans, enabling them to become advisors when we need them. Ambient displays and tangible interfaces suggest the road forward.

All of these new approaches make the design process less cumbersome. At the present time, users struggle to manipulate digital objects though they know how to interact smoothly with physical ones. With the widespread use of TUIs, the boundary begins to blur between digital and physical worlds. When any physical object can be instantly given a digital representation and any digital data may be made evident in the physical world, the two begin to merge. Using computer vision, all objects in the physical world, not just the ones with sensors, can become part of our digital environment. The designer, able to work in either or both, experiences "continuous thought" and produces better design.

Acknowledgements. We thank Daniel Belcher, Randolph Fritz, members of DMG Lab at University of Washington and the colleagues at Georgia Tech for giving us insightful feedback and suggestions.

\section{References}

1. Binder, T., Michelis, G.D., Gervautz, M., Jacucci, G., Matkovic, K., Psik, T., Wagner, I.: Supporting configurability in a mixed-media environment for design students. Personal Ubiquitous Comput. 8(5), 310-325 (2004)

2. Horst, R., Webber, M.: Dilemmas in a General Theory of Planning. In: Policy Sciences, vol. 4, pp. 155-169. Elsevier Scientific, Amsterdam (1973)

3. Archea, J.: Puzzle-making: what architects do when no one is looking. In: Kalay, Y.E. (ed.) Principles of Computer-Aided Design: Computability of Design, pp. 37-52. WileyInterscience, Hoboken (1987)

4. Shneiderman, B.: Direct manipulation: a step beyond programming languages. IEEE Computer 16(8), 57-69 (1983)

5. Hall, R.: Supporting Complexity and Conceptual Design in Modeling Tools, in State of the Art in Computer Graphics: Visualisation and Modeling. In: Rogers, D.F., Earnshaw, R.A. (eds.) 153 - Quotation from R. Sproull, Keynote Speech, SIGGRAPH 1990. Springer, Heidelberg (1991)

6. Ishii, H., Ullmer, B.: Tangible bits: towards seamless interfaces between people, bits and atoms. In: Proc. SIGCHI 1997. ACM Press, New York (1997)

7. Underkoffler, J., Ishii, H.: URP: a luminous-tangible workbench for urban planning and design. In: Proc. SIGCHI 1999, ACM Press, New York (1999)

8. Anderson, D., Frankel, J.L., Marks, J., Agarwala, A., Beardsley, P., Hodgins, J., Leigh, D., Ryall, K., Sullivan, E., Yedidia, J.S.: Tangible interaction + graphical interpretation: a new approach to 3D modeling. In: Proc. 27th Annual Conference on Computer Graphics and Interactive Techniques. ACM Press/Addison-Wesley Publishing Co., New York (2000)

9. Eng, M., Camarata, K., Do, E.Y.-L., Gross, M.D.: FlexM: Designing A Physical Construction Kit for 3d Modeling. In: Akin, O., Krishnamurti, R., Lam, K.P. (eds.) Generative CAD Systems: Proc. GCAD 2004 International Symposium on Generative CAD Systems, Carnegie Mellon University (2004) 
10. Weller, M.P., Do, E.Y.-L., Gross, M.D.: Posey: instrumenting a poseable hub and strut construction toy. In: Proc. 2nd International Conference on Tangible and Embedded Interaction. ACM Press, New York (2008)

11. Song, H., Guimbretière, F., Hu, C., Lipson, H.: ModelCraft: capturing freehand annotations and edits on physical 3D models. In: Proceedings of the 19th Annual ACM Symposium on User Interface Software and Technology, Montreux, Switzerland, pp. 13-22. ACM, New York (2006)

12. HIT Lab. (n.d.). ARToolKit, http: / /www. hitl .washington. edu/artoolkit/ (retrieved March 1, 2009) 